\title{
COMPARISON OF TOPOLOGIES ON FUNCTION SPACES
}

\author{
JAMES R. JACKSON
}

1. Introduction. Let $X$ be a topological space, $Y$ be a metrizable space, and $C$ be the collection of continuous functions on $X$ into $Y$. We shall be interested in two topologies on $C$.

The compact-open or $k$-topology. Given compact subset $K$ of $X$ and open subset $W$ of $Y$, denote by $(K, W)$ the collection of functions $f \in C$ such that $f(K) \subset W$. The assembly of finite intersections of sets $(K, W)$ forms a base for a topology on $C$, called the compactopen topology, or $k$-topology [1]. ${ }^{1}$ Here the restriction to metrizable spaces $Y$ is unnecessary.

The topology of uniform convergence or $d^{*}$-topology. If $Y$ is metrizable, let $d$ be a bounded metric consistent with the topology of $Y$. For instance, if $d^{\prime}$ is an unbounded metric, let

$$
d\left(y_{1}, y_{2}\right)=\min \left\{d^{\prime}\left(y_{1}, y_{2}\right) ; 1\right\}, \quad y_{1}, y_{2} \in Y .
$$

Then for any two elements $f$ and $g$ of $C$, define

$$
d^{*}(f, g)=\sup d(f(x), g(x)) \quad \text { over } X \text {. }
$$

It is easily verified that $d^{*}$ is a metric function, and hence determines a topology on $C$. This topology is called the topology of uniform convergence with respect to $d$, or $d^{*}$-topology [3].

Arens [1] and Fox [2] have shown that the $k$-topology has certain properties which particularly adapt it to the study of various topological problems, particularly those concerned with homotopy theory. But in case $Y$ is metrizable, so that the $d^{*}$-topology can be defined, it is obviously easier to deal with than the $k$-topology. Hence it is of interest to inquire when the two topologies are equivalent.

It is easily shown that if $Y$ is metrizable and $X$ is compact, then the $d^{*}$-topology on $C$ is equivalent to the $k$-topology (see, for instance, [3]). On the other hand, a theorem of Fox [2] implies that if $X$ is a separable metric space which is not locally compact and $Y$ is the real line, then the two topologies are inequivalent. $\mathrm{Hu}$ [3] gives an example due to Liang Ma wherein $X$ is a countable discrete space, and the two topologies are inequivalent. We shall answer in a fairly general way the question of the relationship between these topologies.

Let $Y$ be metrizable. We shall show in $\$ 2$, without further restric-

Received by the editors February 12, 1951.

1 Numbers in brackets refer to the references cited at the end of the paper. 
tion on $X$ or $Y$, that the $d^{*}$-topology is weaker than the $k$-topology (that is, every set open under the $k$-topology is also open under the $d^{*}$-topology). As remarked above, the compactness of $X$ is a sufficient condition for the equivalence of the two topologies. We shall show in $\$ 3$ that if $X$ is completely regular and $Y$ contains a nondegenerate arc, then the compactness of $X$ is also a necessary condition for the equivalence of the $d^{*}$-topology and the $k$-topology.

2. Theorem. If $Y$ is metrizable, then the $d^{*}$-topology is weaker than the k-topology.

We must show that if $U$ is open under the $k$-topology and $f \in U$, then we can find $\epsilon>0$ such that if $d^{*}(f, g)<\epsilon$, then $g \in U$.

By hypothesis, there exist compact subsets of $X, K_{1}, \cdots, K_{n}$, and open subsets of $Y, W_{1}, \cdots, W_{n}$, such that

$$
f \in\left(K_{1}, W_{1}\right) \cap \cdots \cap\left(K_{n}, W_{n}\right) \subset U .
$$

Since each $K_{i}$ is compact, so is each $f\left(K_{i}\right)$. Also, each $f\left(K_{i}\right)$ is disjoint from the corresponding closed set $Y-W_{i}$. But the distance between two disjoint subsets of metric space, one of which is compact and the other closed, is positive. Let $\epsilon$ be a positive number less than the smallest of the distances from the $f\left(K_{i}\right)$ to the corresponding $Y-W_{i}$.

Now suppose $d^{*}(f, g)<\epsilon$. If $x \in K_{i}$, then the distance from $g(x)$ to $f\left(K_{i}\right)$ is less than $\epsilon$, so $g(x) \in W_{i}$. Thus

$$
g \in\left(K_{1}, W_{1}\right) \cap \cdots \cap\left(K_{n}, W_{n}\right) \subset U,
$$

so $g \in U$, as required.

3. Theorem. Let $X$ be a completely regular space, and let $Y$ be a metrizable space containing a nondegenerate arc. Then a necessary condition that the $d^{*}$-topology and the k-topology be equivalent is that $X$ be compact.

Let $\phi: I \rightarrow Y$ define a nondegenerate arc, where $I$ denotes the closed unit interval. Pick $t_{1} \in I$ so $y_{1}=\phi\left(t_{1}\right)$ is different from $y_{0}=\phi(0)$. Choose $\epsilon>0$ so $d\left(y_{0}, y_{1}\right) \geqq \epsilon$. Define $f$ as the function of $C$ which is constantly equal to $y_{0}$.

Suppose the two topologies are equivalent. Then there must be compact subsets of $X, K_{1}, \cdots, K_{n}$, and open subsets of $Y, W_{1}$, $\cdots, W_{n}$, such that

$$
f \in\left(K_{1}, W_{1}\right) \cap \cdots \cap\left(K_{n}, W_{n}\right) \subset S,
$$

where $S$ is the set of functions $g \in C$ such that $d^{*}(f, g)<\epsilon$.

We complete the proof by contradiction. If $X-\left(K_{1} \cup \ldots \cup K_{n}\right)$ 
is empty, then $X$ is the union of a finite number of compact sets, and hence must be compact.

Otherwise, choose $x_{0} \in X-\left(K_{1} \cup \ldots \cup K_{n}\right)$. Since $x_{0}$ and $\left(K_{1} \cup \ldots \cup K_{n}\right)$ are disjoint closed subsets of the completely regular space $X$, there exists a continuous function $\theta: X \rightarrow I$ with $\theta(x)=0$ on $\left(K_{1} \cup \cdots \cup K_{n}\right)$ and $\theta\left(x_{0}\right)=t_{1}$. Define $g: X \rightarrow Y$ as the product $\phi \theta ; g(x)=\phi(\theta(x))$. It is obvious that $g \in C$. Moreover, for $x \in K_{i}$, we have that $g(x)=\phi(\theta(x))=\phi(0)=y_{0} \in W_{i}$; so $g\left(K_{i}\right) \subset W_{i}$, and $g$ $\in\left(K_{1}, W_{1}\right) \cap \cdots \cap\left(K_{n}, W_{n}\right)$.

On the other hand,

$$
\begin{aligned}
d^{*}(f, g) \geqq d\left(f\left(x_{0}\right), g\left(x_{0}\right)\right) & =d\left(y_{0}, \phi\left(\theta\left(x_{0}\right)\right)\right) \\
& =d\left(y_{0}, \phi\left(t_{1}\right)\right) \\
& =d\left(y_{0}, y_{1}\right) \geqq \epsilon .
\end{aligned}
$$

Hence $g \notin S$. This contradiction completes the proof.

4. A generalization. If instead of requiring $Y$ to be metrizable, we insist only that it be a uniform space, a topology of uniform convergence can be defined on $C$. In this case the compactness of $X$ is a sufficient condition for the equivalence of the topology of uniform convergence and the $k$-topology, the obvious analogue of Theorem 2 holds without further restriction, and the analogue of Theorem 3 holds if we require that $Y$ have a separated uniform structure. The proofs are not essentially different from those above, but are somewhat longer.

\section{REFERENCES}

1. R. Arens, A topology for spaces of transformations, Ann. of Math. vol. 47 (1946) pp. $480-495$.

2. R. Fox, On topologies for function spaces, Bull. Amer. Math. Soc. vol. 51 (1945) pp. 429-432.

3. S. T. Hu, Homotopy theory, vol. 1 (Dittoed typescript prepared at Tulane University under the auspices of the Office of Naval Research), 1950.

University of California, Los Angeles 